\title{
Termoterapia transpupilar como opção terapêutica para hemangioma circunscrito de coróide - Relato de caso
}

\author{
Transpupillary thermotherapy as therapeutic option for circumscribed choroidal \\ hemangioma-Case report
}

Jaime Roizenblatt ${ }^{1}$

Alexandre Antonio Marques Rosa ${ }^{2}$

\footnotetext{
${ }^{1}$ Médico Assistente da Clínica Oftalmológica do Hospital das Clínicas da Faculdade de Medicina da Universidade de São Paulo.

${ }^{2}$ Médico Estagiário do Setor de Retina do Departamento de Oftalmologia do Hospital das Clínicas da Faculdade de Medicina da Universidade de São Paulo.

Endereço para correspondência: Av. Angélica, 1045 $5^{\circ}$ andar - São Paulo (SP) CEP 01227-100.

E-mail: jroizenb@usp.br

Nota Editorial: Pela análise deste trabalho e por sua anuência sobre a divulgação desta nota, agradecemos ao Dr. Michel Eid Farah Neto.

Recebido para publicação em 22.05.2001

Aceito para publicação em 16.01.2002
}

\section{RESUMO}

Os autores descrevem um caso de hemangioma circunscrito de coróide, com descolamento de retina associado, tratado por meio de termoterapia transpupilar com laser de diodo. Os parâmetros utilizados foram: $900 \mathrm{~mW}$, mira de 4,2 mm, tempo de 3 minutos, lente QuadrAspheric $\left(\operatorname{Volk}^{\circledR}\right)$ com fator de magnificação de 1,97 . Um mês após o tratamento ocorreu redução significativa do hemangioma, com recuperação integral da visão deste olho. São feitas considerações a respeito do método, o qual mostra-se promissor no tratamento destes tumores vasculares.

Descritores: Hipertermia induzida; Neoplasias da coroideterapia; Hemangioma/ terapia; Descolamento retiniano; Lasers/uso terapêutico

\section{INTRODUÇÃO}

Hemangioma de coróide é um tumor vascular benigno composto de grandes vasos dilatados da coróide. Pode ocorrer como um tumor circunscrito, sem associações extra-oculares, ou de forma difusa, o qual está associado com nevus flammeus facial ou variações da síndrome de SturgeWeber ${ }^{(1)}$.

Oftalmoscopicamente, o hemangioma de coróide circunscrito aparece como um tumor elevado vermelho-alaranjado localizado nas camadas mais profundas da retina. Pode induzir o aparecimento de um descolamento de retina exsudativo secundário, causando baixa de visão, quando se estende à área macular ${ }^{(2)}$.

O padrão angiográfico revela um enchimento precoce do tumor, simultâneo ao enchimento coróideo, com hiperfluorescência tardia.

A ultrassonografia B mostra uma lesão sólida, com alta refletividade interna no modo A. O diagnóstico diferencial deve ser feito com melanoma de coróide amelanótico e tumor metastático.

$\mathrm{Na}$ forma difusa, ocorre um espessamento coroidiano difuso dando ao fundo de olho uma coloração vermelha-escura, fazendo com que a lesão passe despercebida se a coloração do fundo de olho não for comparada com o olho contralateral. A compressão digital do bulbo ocular também ajuda no diagnóstico, pois nesta situação a lesão empalidece.

Os casos assintomáticos devem ser observados periodicamente, pois, na maioria dos casos, há crescimento da lesão com o passar do tempo. Aqueles que apresentam descolamento de retina secundário podem ser tratados com fotocoagulação para eliminar o líquido subretiniano ${ }^{(2-3)}$, a qual nem sempre é eficaz. Os tumores grandes com descolamentos extensos podem às vezes ser controlados com radioterapia ${ }^{(4)}$, porém esta freqüente- 
mente resulta em efeitos colaterais graves para a retina e tecidos circunjacentes.

Termoterapia transpupilar (TTT) é uma modalidade de tratamento que utiliza um laser de diodo modificado, com comprimento de onda de $810 \mathrm{~nm}$, para produzir um aquecimento localizado e uniforme com subseqüente obliteração dos vasos mal formados e redução no tamanho do tumor ${ }^{(5)}$.

A termoterapia transpupilar foi primeiramente descrita por Oosterhuis et al. ${ }^{(6)}$ em 1995, quando foi usada como coadjuvante à radioterapia no tratamento de melanomas de coróide, durante e após o tratamento com placa, em casos nos quais a regressão do tumor foi insuficiente.

Shields et al. estudaram a efetividade da TTT isoladamente em pequenos melanomas de coróide ${ }^{(5)}$. Estes estudos demonstraram como a anatomia do olho permite a transmissão da luz e facilita o uso da TTT no tratamento de patologias oculares do segmento posterior.

Hipertermia é definida como uma elevação da temperatura acima da temperatura normal. Em contraste à fotocoagulação a laser, na hipertermia os efeitos produzidos pela coagulação estão ausentes, otimizando a penetração do calor quando realizada apropriadamente ${ }^{(7-8)}$. Isto se deve ao fato que as camadas superficiais da retina não são afetadas na TTT, permitindo que a energia luminosa chegue até as camadas mais profundas.

Othmane et al. ${ }^{(9)}$ demonstraram um caso de hemangioma de coróide tratado por meio da termoterapia transpupilar que apresentou redução na espessura do tumor após a aplicação do laser.

Existem relatos na literatura demonstrando ótimos resultados de pacientes com hemangiomas de coróide circunscritos e descolamento de retina secundário tratados com termoterapia transpupilar ${ }^{(10-12)}$

\section{RELATO DE CASO}

G.V.P.V.; 48 anos, masculino, pardo, com história de baixa de acuidade visual progressiva em olho direito a um mês. Ao exame ocular, apresentava acuidade visual com a melhor correção de 20/50 e 20/20 no olho direito e esquerdo, respectivamente. À fundoscopia do olho direito, observava-se uma massa coroidal vermelho-alaranjada, bem circunscrita, arredondada, apresentando cerca de 7 diâmetros papilares no seu maior diâmetro (cerca de $8 \mathrm{~mm}$ ), localizada nasalmente ao disco óptico, e a presença de um descolamento seroso de retina envolvendo a mácula (Figura 1).

A angiografia fluorescente mostrava uma hiperfluorescência pré-arterial com vazamento tardio (Figura 2).

Ecograficamente no modo B apresentava um descolamento de retina seroso, associado com uma massa sólida próxima ao nervo óptico, com aproximadamente $4 \mathrm{~mm}$ de espessura, e alta refletividade interna no modo A (Figura 3), compatível com o diagnóstico de hemangioma circunscrito de coróide.

A lesão foi tratada com laser de diodo infravermelho (TTT

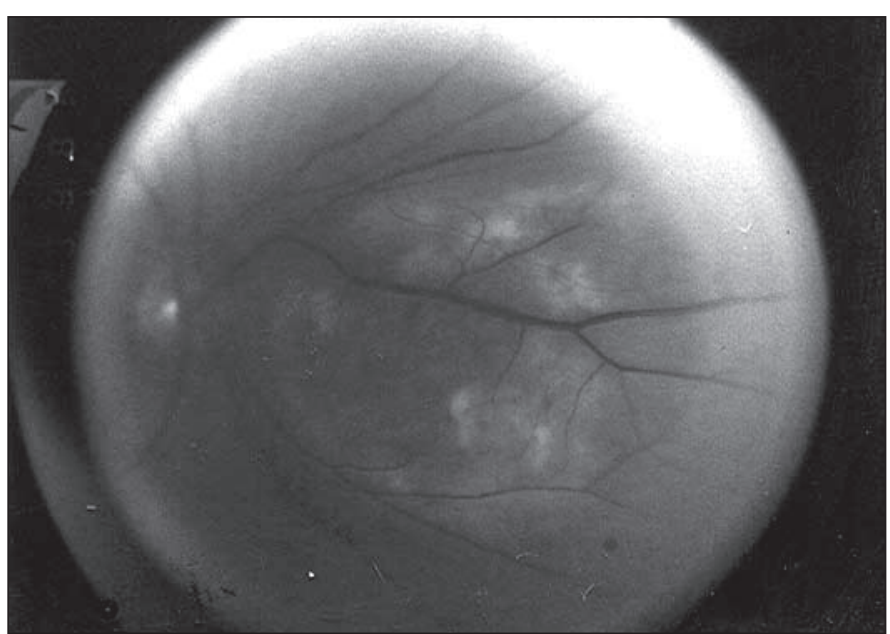

Figura 1 - Retinografia do olho direito. Observa-se uma massa coroidal vermelho-alaranjada, correspondente a um hemangioma circunscrito de coróide, localizada nasalmente ao disco óptico

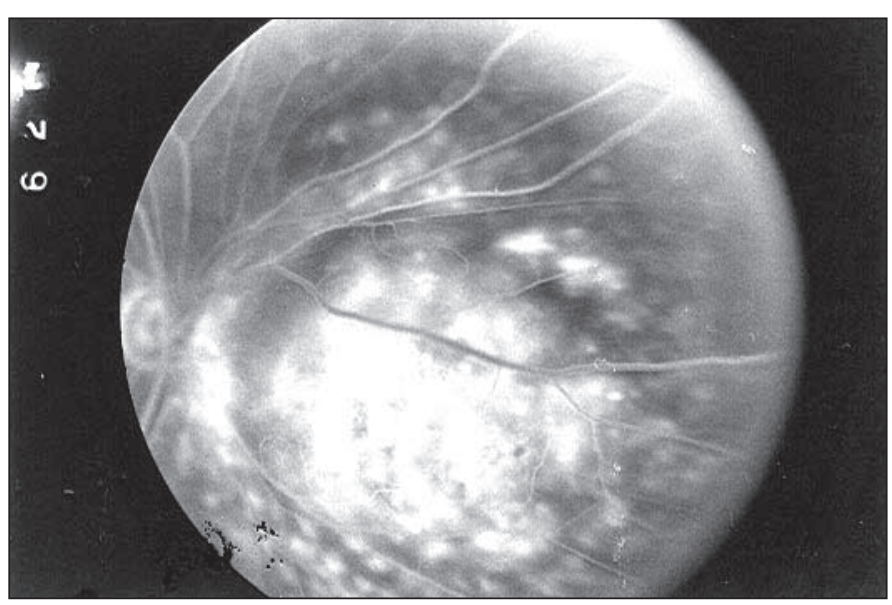

Figura 2 - Retinografia fluorescente do olho direito. Observa-se impregnação do corante na lesão

$1500^{\circledR}-$ Opto $^{\circledR}$ ) com uma potência de $900 \mathrm{~mW} ; 4,2 \mathrm{~mm}$ de mira, por cerca 3 minutos, usando-se uma lente de contato com fator de magnificação de 1,97 (QuadrAspheric ${ }^{\circledR}$ - Volk ${ }^{\circledR}$ ) que resultou numa mira com $8,3 \mathrm{~mm}$ ao nível da retina, que praticamente circunscrevia todo o tumor.

O tempo de aplicação do laser foi determinado pela própria reação tecidual, pois ao final de 3 minutos começou a ocorrer uma discreta mudança da coloração do hemangioma, sendo o tratamento interrompido neste ponto.

Um mês depois se observou, oftalmoscopicamente, uma redução significativa do tamanho da lesão do olho direito, com absorção do líquido subretiniano (Figura 4). Este fato era também evidenciável na retinografia fluorescente (Figura 5) e na ecografia (Figura 6). Houve uma melhora significativa da acuidade visual, retornando para 20/20. O seguimento do paciente já dura 5 meses, sendo que no local do hemangioma observa-se uma cicatriz pigmentada e plana, com discreta elevação junto do disco óptico. 


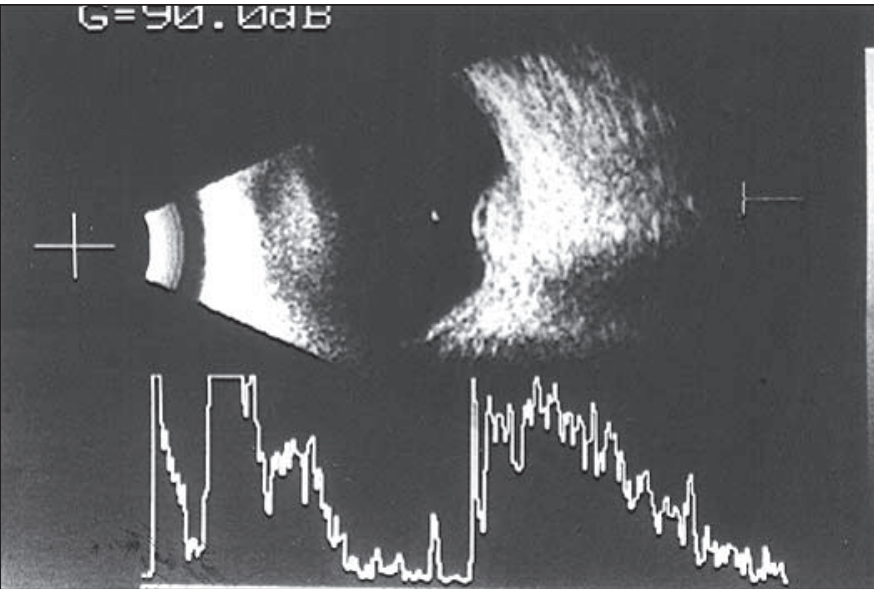

Figura 3 - Ecografia do olho direito. No modo B, observa-se um descolamento de retina seroso, associado com uma massa sólida próxima ao nervo óptico, com alta refletividade interna no modo $A$, compatível com o diagnóstico de hemangioma circunscrito de coróide

\section{DISCUSSÃO}

Desde 1860, a hipertermia tem sido usada como uma modalidade terapêutica para tumores. Neste tempo, foi notado que sarcomas faciais regrediam após quadros febris prolonga$\operatorname{dos}^{(13)}$. Os estudos iniciais em seres humanos foram realizados em tumores cutâneos como carcinomas de células escamosas e melanomas malignos ${ }^{(14-16)}$.

Em contraste com a fotocoagulação a laser, na hipertermia, os efeitos produzidos pela coagulação das proteínas teciduais estão ausentes, otimizando a penetração do calor. A penetração do calor também é otimizada pelo tempo de exposição, diâmetro do feixe e comprimento de onda. Exposições de um minuto ou mais com um feixe de vários milímetros de diâmetro e comprimento de onda entre 700 e 900 nm são necessárias ${ }^{(8)}$. Irradiação próxima do espectro do infravermelho é a ideal para o uso na termoterapia transpupilar, pois a penetração tecidual é alta e a absorção pelos meios oculares é mínima ${ }^{(17)}$. Pelo

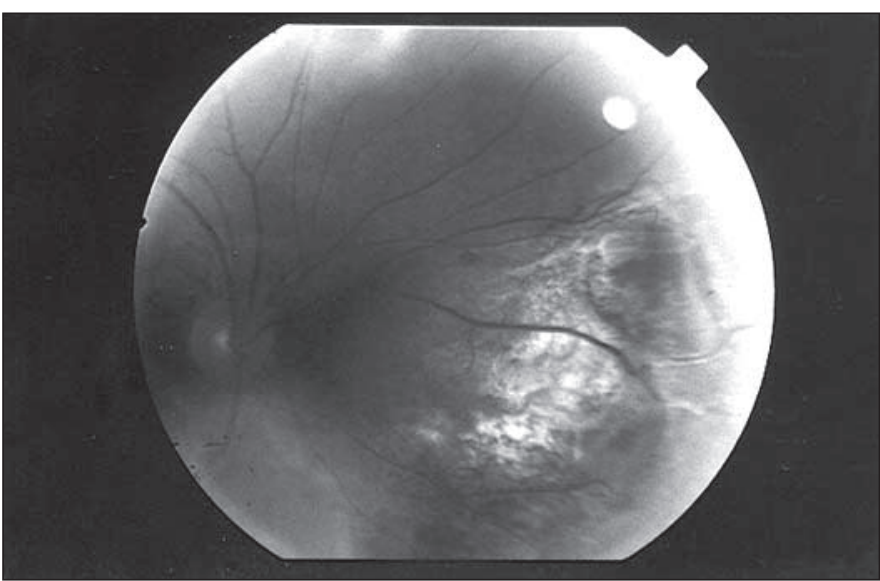

Figura 4 - Retinografia do olho direito pós-tratamento com TTT. Notase uma redução no tamanho da lesão vascular

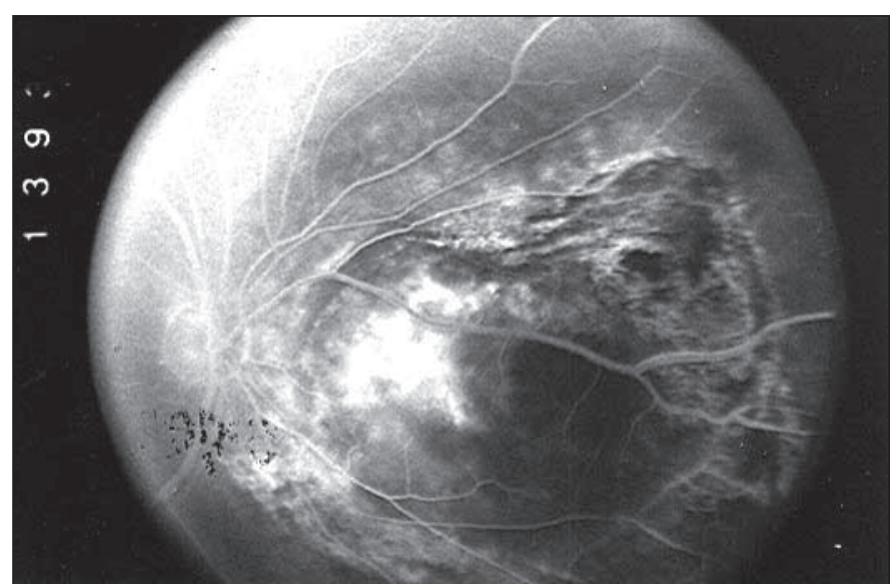

Figura 5 - Retinografia fluorescente do olho direito. Nota-se uma diminuição na impregnação de contraste pelo hemangioma de coróide

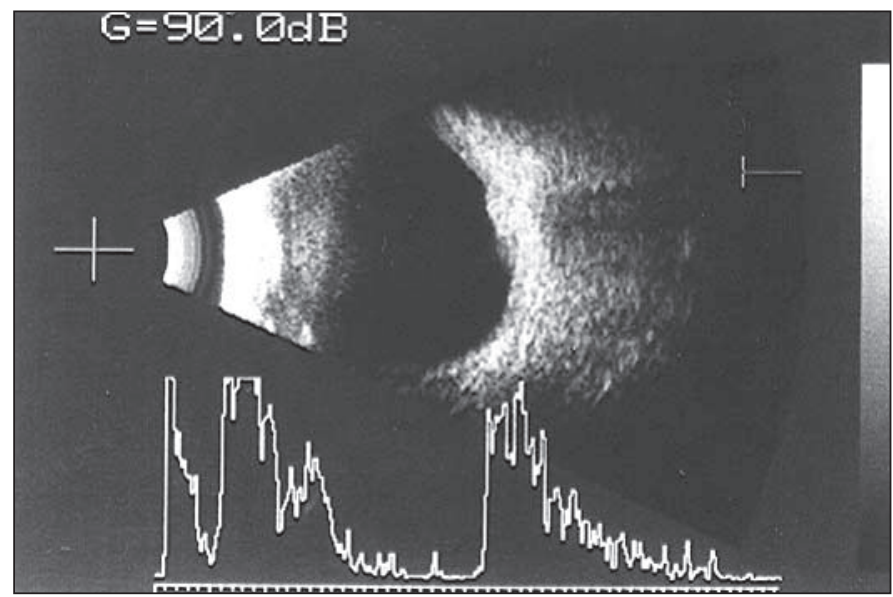

Figura 6 - Ecografia do olho direito. Observa-se uma nítida redução no tamanho da lesão tumoral

aumento no diâmetro do feixe, a penetração de calor no tecido alvo é aumentada e a dissipação de calor nos tecidos adjacentes é diminuída. Os níveis diminuídos de calor proporcionados pelo TTT, em comparação com a fotocoagulação a laser, causam dano tecidual colateral mínimo à retina adjacente.

Estudos histológicos de melanomas de coróide tratados com TTT mostram extensa trombose dos vasos tumorais após o tratamento ${ }^{(18)}$. Suspeitamos que algo análogo possa ocorrer nos hemangiomas de coróide, levando à regressão do tumor.

Baseados nos relatos dos autores que descreveram o uso da termoterapia transpupilar para tratamento dos hemangiomas de coróide ${ }^{(9-12)}$ e na evolução observada no presente caso, pode-se dizer que as vantagens da termoterapia transpupilar, quando comparada à fotocoagulação convencional, são: o menor número de aplicações com o laser de diodo para obter o desaparecimento da lesão, recuperação visual mais rápida, especialmente nos casos em que há descolamento de retina secundário e eficácia da TTT mesmo naqueles casos em que a fotocoagulação convencional com laser de argônio foi infrutífera. 
Os níveis de energia utilizados por outros autores para tratar hemangiomas de coróide tem sido variáveis. Othmane et al. ${ }^{(9)}$ descreveram o uso de $550 \mathrm{~mW}$, mira de 3 milímetros, durante 10 minutos, para tratar um paciente com hemangioma localizado de coróide. Garcia-Arumi et al. ${ }^{(10)}$ usaram entre 800 e $1200 \mathrm{~mW}, 3$ a 6 minutos de exposição, trabalhando com miras de 2 a $3 \mathrm{~mm}$ quando trataram 8 olhos de 8 pacientes com hemangiomas de coróide de diferentes dimensões. Rapizzi et al. ${ }^{(12)}$ usaram $510 \mathrm{~mW}$, mira de $2 \mathrm{~mm}$ e 5 minutos de aplicação. Todos estes autores interrompiam o tratamento quando a lesão começava a mudar de coloração ou ficava levemente esbranquiçada.

Os níveis de energia $(\mathrm{mW})$ utilizados no presente caso estão dentro da mesma faixa utilizada pelos outros autores, ressalvando-se o tempo de aplicação mais curto. Uma possível explicação é o tamanho da mira, que por ser maior do que a utilizada pelos outros autores, permitiu possivelmente uma penetração mais eficaz de energia no tecido alvo. Como dito anteriormente, quanto maior a mira, menor é a dissipação de calor nos tecidos adjacentes à lesão. A utilização de miras maiores do que $3 \mathrm{~mm}$ para tratamentos que utilizam o laser de diodo foi proposta por um dos autores (JR - comunicação pessoal a $\mathrm{Opto}^{\circledR}$ ) e o presente caso ilustra bem a utilidade desta modificação.

Várias modalidades terapêuticas já foram empregadas para tratamento dos hemangiomas como laser de argônio, placas de braquiterapia, radiação externa, radiação por feixe de prótons ${ }^{(2)}$. Todas esbarram com freqüência maior ou menor em taxas de insucesso elevadas e efeitos colaterais importantes. Portanto, a chegada de alternativas terapêuticas, como a termoterapia transpupilar e a terapia fotodinâmica, que pelo menos inicialmente, parecem apresentar maior margem de sucesso e menor incidência de efeitos indesejáveis, são muito bem-vindas.

Os parâmetros ideais para TTT ainda estão para serem elucidados. Estudos randomizados e prospectivos usando um grande número de pacientes determinarão o papel da TTT no tratamento dos hemangiomas de coróide, mas mostra-se como uma importante modalidade de tratamento a ser considerada nestes pacientes.

\section{ABSTRACT}

The authors describe a case of a circumscribed choroidal hemangioma with secondary retinal detachment treated with transpupillary thermotherapy using diode laser. The settings were: $900 \mathrm{~mW}$ power, $4.2 \mathrm{~mm}$ spot, for 3 minutes, QuadrAspheric $^{\circledR}$ lens $\left(V_{o l k}{ }^{\circledR}\right)$ with a magnification factor of 1.97 . One month later, there was a significant shrinkage of the hemangioma with full recovery of vision in this eye. The authors discuss some aspects of this method, which seems promising in treatment of this vascular tumors.

Keywords: Induced hyperthermia; Choroid neoplasms/ therapy; Hemangioma therapy; Retinal detachment; Lasers/ therapeutic uses

\section{REFERÊNCIAS}

1. Shields JA, Shields CL. Vascular tumors of the uvea. In: Shields JA, Shields CL. Intraocular tumors: a text and atlas. Philadelphia.: WB Saunders; 1991. p.239-59.

2. Anand R, Augsburger JJ, Shields JA. Circumscribed choroidal hemangioma. Arch Ophthalmol 1989;107:1338-42.

3. Shields JA. The expanding role of laser photocoagulation for intraocular tumors: The 1993 H. Christian Zweng Memorial Lecture. Retina 1994;14:310-22.

4. Schilling H, Sauerwein W, Lommatzsch A, Friedrichs W, Brylak S, Bornfeld $\mathrm{N}$, et al. Long-term results after low-dose ocular irradiation for choroidal hemangiomas. [commented on Br J Ophthalmol 1997;81:258]. Br J Ophthalmol 1997;81:267-73.

5. Shields CL, Shields JA, Cater J, Lois N, Edelstein C, Gunduz K, Mercado G. Transpupillary thermotherapy for choroidal melanoma: tumor control and visual results of 100 consecutive cases. Ophthalmology 1998;105:581-90.

6. Oosterhuis JA, Journee-de Korver HG, Kakebeeke-Kemme HM, Bleeker JC. Transpupillary thermotherapy in choroidal melanomas. Arch Ophthalmol 1995; 113:315-21.

7. Burgess SEP, Chang S, Svitra P, Driller J, Lizzi FL, Coleman DJ. Effect of hyperthermia on experimental choroidal melanoma. Br J Ophthalmol 1985;69:854-60.

8. Coleman DJ, Silverman RH, Iwamoto T, Lizzi FL, Rondeau MJ, Driller J, et al. Histopathologic effects of ultrasonically induced hyperthermia in intraocular malignant melanoma. Ophthalmology 1988;95:970-81.

9. Othamne IS, Shields CL, Shields JA, Gunduz K, Mercado G. Circumscribed choroidal hemangioma managed by transpupillary thermotherapy. Arch Ophthalmol 1999;117:136-7.

10. Garcia-Arumi J, Ramsay LS, Guraya BC. Transpupillary thermotherapy for circumscribed choroidal hemangiomas. Ophthalmology 2000;107:351-6. discussion p.357.

11. Kamal A, Watts AR, Rennie IG. Indocyanine green enhanced transpupillary thermotherapy of circumscribed choroidal haemangioma. Eye 2000; $14 \mathrm{Pt}$ 5:701-5.

12. Rapizzi E, Grizzard WS, Capone A. Transpupillary thermotherapy in the management of circumscribed choroidal hemangioma. Am J Ophthalmol 1999;127:481-2.

13. Finger PT, Packer S, Svitra PP, Paglione RW, Chess J, Albert DM. Hyperthermic treatment of intraocular tumors. Arch Ophthalmol 1984;102:1477-81.

14. Kim JH, Hahn EW, Tokita N. Combination hyperthermia and radiation therapy for cutaneous malignant melanoma. Cancer 1978;41:2143-8.

15. Kim JH, Hahn EW, Tokita N, Nisce LZ. Local tumor hyperthermia in combination with radiation therapy. 1. Malignant cutaneous lesions. Cancer 1977;40:161-9

16. Overgaard J, Overgaard M. Hyperthermia as an adjuvant to radiotherapy in the treatment of malignant melanoma. Int J Hyperthermia 1987;3:483-501.

17. Svaasand LO, Boerslid T, Oeverraasssen M. Thermal and optical properties of living tissue: application to laser-induced hyperthermia. Lasers Surg Med 1985;5:589-602.

18. Journee-de Korver JG, Oosterhuis JA, de Wolff-Rouendaal D, Kemme H. Histopathological findings in human choroidal melanomas after transpupillary thermotherapy. Br J Ophthalmol 1997;81:234-9. 